\title{
Simplicity as a principle in tactual form perception
}

JON E. ROECKELEIN, Arizona State University, Tempe, Ariz.' 85281

Simplicity was operationally defined using measurable characteristics of stimuli. High correlations between these objective models and S's rank ordering of stimuli along a simplicity-complexity continuum permitted the application of the "simplicity" principle to active touch form perception. The comparison of two methods of stimulus presentation for active touch showed that a "whole" method using simultaneous presentation of stimuli was superior to a "part" method using paired comparisons. The information processing capacity of $S$ for tasks employing active touch was also discussed.

In developing his notions regarding "higher-order" variables in visual perception, Hochberg (1965) discussed the principle of organization and noted that the Gestalt theorists used ambiguous patterns to derive numerous laws of perception. Most of these laws were summarized under the principle of "simplicity." Other predictive laws of visual perception which have developed from the Gestalt tradition (e.g., shape and form) are based upon objective measures of simplicity such as "number of angles," "average number of different angles," and "number of continuous lines" (Hochberg, 1965, p. 89).

The similarity and differences between the two senses, touch and vision, within a passive-active dimension were described by Gibson (1962) who concluded that the two senses, in some respects, "seem to register the same information and to yield the same phenomenal experiences [p. 490]." Other studies have investigated the relationships between touch and vision on various bases such as exposure time (Lobb, 1965), illusions and reversal figures (Shopland \& Gregory, 1964), and conflict between the senses (Rock \& Harris, 1967; Rock \& Victor, 1964). Typically, these studies have employed active touch methodologies (Gibson, 1962; 1963). However, the possible confounding effects provided by visual cues of Ss' tasks in these studies have left statements and conclusions concerning active touch, per se, somewhat equivocal.

The two objectives of the present study were: (a) to explore the extent to which tactual form perception via active touch is governed by a principle that holds for visual perception, viz., the principle of simplicity; and (b) to collect data concerning Ss' information-processing capacities via active touch along a simplicity-complexity stimulus continuum. The first purpose was developed using an "objective model" criterion applied to stimuli (Hochberg, 1965; Gibson, 1962). The second purpose involved the use of two different methods of stimulus presentation: all materials presented simultaneously ("Whole" method), and presentation of only two stimuli at a time ("Part" method).

\section{Stimulus Objects}

\section{METHOD}

Two sets of stimuli were used in two different tasks. One set (A) consisted of six designs adapted from Gibson $(1962$, p. 486); each design was cut from a 41/2-in. $\times 41 / 2$-in. $x 1 / 8$-in. hard-rubber tile square. Another set (B) consisted of three designs adapted from Hochberg (1965, p. 90) and were constructed of wooden dowels mounted on wooden blocks. Both sets of stimuli differed along a simplicity-complexity continuum: For Set A, the criterion was number of protuberances or points; for Set B, the criterion was number of interior angles, average number of different angles, and number of continuous lines. Numbers from 1 through 6 were assigned to the "objective" model of Set $\mathrm{A}$; numbers from 1 through 3 were assigned to the "objective" model of Set B. In all cases, the lowest number was assigned to the simplest design and the highest number was assigned to the most complex design.

Procedure

Twenty students in an undergraduate psychology course were randomly placed in one of two groups. Group 1 was given a paired comparison method of stimulus presentation ("Part" method); Group 2 was given a rank order method of presentation ("Whole" method). A large curtain through which $S$ put his hands and forearms was hung between $S$ and $E$ to prevent $\mathbf{S}$ from seeing the stimulus objects. Each $\mathbf{S}$ in Group 1 was informed that pairs of stimuli would be presented to him and his task was to tell $\mathrm{E}$ which of the two stimuli was the "simplest." Each $\mathbf{S}$ in Group 2 was instructed to rank order the stimuli which he received by placing them in a line in front of him on the table from the "simplest" to the "most complex" from his left to his right. Stimulus presentation order and task order were randomly determined. There were two tasks for each group: Task A, stimulus Set $A$ used; and Task B, stimulus Set B used. Each S was allowed to take as much time as he needed to compare or to rank order the stimuli. Observations and qualitative records were made of S's hand movements and verbalizations during the experiment.

\section{RESULTS}

Data for Groups 1 and 2 were separately entered on 6 by 6 matrix tables for Task A and 3 by 3 tables for Task B. Two rank order scores for each group were then computed, one score for the six objects in Task A and one score for the three objects in Task B. Per cent of time that one of the two stimuli was judged as "simplest" was the criterion for cell entry. Using the two "objective models" as idealized groups of scores, the correlation measure tho $(p)$ was computed for each method of stimulus presentation for each task. Table 1 shows the groups' rank orderings of stimuli against the objective models, and the corresponding value of $p$ for the two methods of stimulus presentation.

The term "error" in Table 1 refers to "errors in judgment" regarding the simplicity-complexity continuum and is the per cent of time that ratings of objectively simple stimuli were judged as complex.

\section{DISCUSSION}

The results of this study point up two facts concerning active touch: (a) The principle of simplicity can feasibly be applied to an analysis of tactual form perception; and (b) the capacity for information processing for tactual form perception is mensurable. In the first instance, it is evident that the employment of an "objective model" criterion based upon the measurable physical characteristics of the task

Table 1

The Rank Order of Stimulus Materials via Active Touch Along a Simplicity-Complexity Continuum Using Two Methods of Presentation

\begin{tabular}{|c|c|c|}
\hline \multirow{2}{*}{$\begin{array}{c}\text { Method of Stimulus } \\
\text { Presentation }\end{array}$} & \multicolumn{2}{|c|}{ Assigned Rank } \\
\hline & Task A Stimuli & Task B Stimuli \\
\hline $\begin{array}{l}\text { "Part" method } \\
\text { (Group 1) }\end{array}$ & $\begin{array}{c}2-3-1-4-5-6 \\
p=.83 \\
\text { "error" }=18.1 \%\end{array}$ & $\begin{array}{c}1-2-3 \\
p=1.00 \\
\text { "error" }=0.7 \%\end{array}$ \\
\hline $\begin{array}{l}\text { "Whole" method } \\
\text { (Group 2) }\end{array}$ & $\begin{array}{c}1-2-3-4-5-6 \\
p=1.00 \\
\text { "error" }=10.7 \%\end{array}$ & $\begin{array}{c}1-2-3 \\
p=1.00 \\
\text { "error" }=1.3 \%\end{array}$ \\
\hline
\end{tabular}

Note-Read number sequences in cells from left to right as "simple to complex." 
stimulus objects (i.e., number of angles, number of protuberances, etc.) was powerful enough to afford an operational definition of simplicity for active touch, and also to permit the construction of a simplicity-complexity continuum along which Ss could rank order stimuli. In the second instance, the addition of stimulus elements to a group of stimuli which must be rank ordered via active touch imposes an error effect upon correct sequential ordering of stimuli which is analogous to information-processing capacities in other senses such as vision, audition, taste and smell (e.g., Miller, 1956). This latter point can be seen from Table 1 by comparing the "Part" method with the "Whole" method as well as comparing the large number of stimuli in Task $A$ with the small number of stimuli in Task B. The trend is clear that for active touch it is easier to rank order stimuli along a simplicity-complexity continuum when the materials are presented all at once, rather than piecemeal (cf. "massed scanning" vs "spaced scanning" of Lobb \& Friend, 1967). However, it is not as clear why Ss made more errors with the "Whole" method than with the "Part" method for the stimuli used in Task B. While both Groups 1 and 2 correctly rank ordered the stimuli in Task B, Group 1 incorrectly judged the "complex" stimuli as "simple" more often than did Group 2 $(1.3 \%$ vs $0.7 \%)$. Perhaps the reasons for this discrepancy can be found from an analysis of the stimuli themselves as well as describing some of Ss' hand movements and verbalizations during Task B. The stimuli or designs used in Task B were originally intended to be used as materials for a haptic illusion study employing active touch alone, but a pilot study revealed that the particular illusion chosen (a Necker cube) and the subsequently constructed stimulus objects were not satisfactory for that purpose (cf. Shopland \& Gregory, 1964). However, the stimuli did contain objectively variable, and measurably different, physical characteristics and were chosen as a partial operational definition of simplicity for the present study. An analysis of Ss' hand movements showed that, on the whole, Ss took more time to feel and handle the stimuli in Task B than in Task A, although there were more objects in A than in B. Also, some Ss indicated via verbalizations that they wanted to give the rating of "Same" to stimuli used in Task B; however, when forced to choose and rank stimuli, they usually made the correct choices. Thus, to a small degree, the "absolute" complexity of the stimuli used in Task B worked against the effect of the two methods of stimulus presentation for that task.

The results of this study also point to the need for further research concerning information processing and the "channel capacity" for the sense of active touch. The highest number of stimuli used here was six, but it is interesting to speculate upon the effects of using larger numbers of elements or longer sequences of stimuli; perhaps Miller's (1956) "magic number seven" will also apply for active touch.

\section{REFERENCES}

GIBSON, J. J. Observations on active touch. Psychological Review, 1962 $69,477-491$

GIBSON, J. J. The useful dimensions of sensitivity. American Psy chologist, 1963, 18, 1-15.

HOCHBERG, J. Perception. New Jersey: Prentice-Hall, 1965.

LOBB, $\mathrm{H}$. Vision versus touch in form discrimination. Canadian Journal of Psychology, 1965, 19, 175-187.

LOBB, H., \& FRIEND, R. Tactual form discrimination with varying size and duration of exposure. Psychonomic Science, -1967, 7, 415-416.

MILLER, G. A. The magical number seven, plus or minus two: Some limits on our capacity for processing information. Psychological Review, 1956, 63, 81-97.

ROCK, I., \& HARRIS, C. S. Vision and touch. Scientific American, $1967,216,96-104$.

ROCK, 1., \& VICTOR, J. Vision and touch: An experimentally created conflict between the two senses. Science, 1964, 143, 594-596.

SHOPLAND, C., \& GREGORY, R. The effect of touch on a visually ambiguous three-dimensional figure. Quarterly Journal of Experimental Psychology, 1964, 16, 66-70.

NOTE

1. The author wishes to thank James E. Scoresby for providing subjects and Joyce E. Pattison for her assistance in the preparation and critical reading of the manuscript. 